\title{
Using a Bayesian approach to evaluate the accuracy of economies of scope: Examples from Australian public universities
}

\author{
$\underline{\text { L.-C. Zhang }}^{\text {a }}$, J.-J. Syu ${ }^{\text {b }}$ and A.C. Worthington ${ }^{\text {a }}$ \\ ${ }^{a}$ Department of Accounting, Finance and Economics, Griffith Business School, Griffith University, \\ Australia; ${ }^{b}$ School of Population Health, University of Queensland, Australia \\ Email: liang-cheng.zhang@griffithuni.edu.au.
}

\begin{abstract}
The point estimate of economies of scope is a nonlinear combination of estimated coefficients from an empirical model. This estimate usually involves out-of-sample predictions when calculating the separate costs (as a part of the calculation of economies of scope). These difficulties make it hard to give the precise prediction and to calculate the standard deviation of this estimate along with its confidence intervals. In this paper, we demonstrate methods for constructing the confidence interval for economies of scope to allow researchers to draw inferences from estimated economies of scope. We review the common approaches such as delta method or bootstrap adopted by previous studies. In contrast to the above approximation methods, this study also proposes an alternative method, Bayesian approach, to produce full predictive distribution for this measure with the posterior distribution. To demonstrate these three approaches, we use a balanced panel data including 37 Australian public universities over the period 2003-12. Our Bayesian approach uses a quadratic cost function with two outputs. Estimates of economies of scope will be calculated with the sample data and estimated parameters from the model.
\end{abstract}

Keywords: Bayesian inference, Delta method, bootstrap, economies of scope, confidence interval 
Zhang et al, Using a Bayesian approach to evaluate the accuracy of economies of scope: Examples from Australian public universities

\section{INTRODUCTION}

Single-product firms that specialize in the production of a single output used to be the concentration of economic analysis. However, the discussion of structure modification of a firm is, therefore, limited in quantities of that single output. To consider the possibility of cost economies from expanding the scope (instead of scale) of the firm's operation, Panzar \& Willig (1981) coined the term "economies of scope". If economies of scope exist, production of a bundle of $\mathrm{n}$ products by one firm will be less costly than by producing the same bundle separately by $\mathrm{n}$ firms. In this case, a single-product firm could save costs by merely diversifying its product line to become a multi-product firm.

So far, the theory of economies of scope have been widely applied to different industries and have played a critical role in evaluating the potential economic benefits from firms' growth. For examples, in the energy industry, a water only company could vertically integrate with sewerage services or horizontally integrate with electricity or gas services based on whether there are economies of scope between water provided services and other services (Saal, Arocena, Maziotis, \& Triebs, 2013). In the bank industry, the existence of economies of scope has been considered an important motivation for bank mergers and acquisitions (Delgado, Parmeter, Hartarska, \& Mersland, 2015). Even in not-for-profit industries such as higher education (Worthington \& Higgs, 2011), health care (Carey, Burgess, \& Young, 2015), and agriculture (Ohe \& Kurihara, 2013), there have also been considerable interests in applying this estimate.

Despite the influence of economies of scope for the firm and industry structure modifications, its estimation, however, is not build on a complete estimation process. Several review articles (Meyer, 2012; Saal et al., 2013) have shown that there are at least two steps to gain the estimate: estimate the cost function with a proper functional form and then calculate the point estimate of economies of scope that are functions of cost function parameter estimates. Their investigations show that most studies inference the existence of economies of scope only by whether a point estimate is larger than zero. Specifically, the degree of economies of scope is estimated by simply plugging in sample data into the estimated cost function without considering its standard error. Therefore, intervals for this estimate could not be constructed, and we have little confidence in it. In other words, the existence of economies of scope is inferred purely by plug-in procedure and it neglects uncertainty over estimates of economies of scope as well as the uncertainty of the cost function coefficients. Therefore, intervals could not be constructed, and we have little confidence in this estimate.

In order to make inference about whether there are economies of scope, calculating its point estimate is not enough. Its confidence interval should be estimated as well. Two approximation methods were used to obtain the confidence interval of economies of scope: delta method and bootstrap. The former method approximates standard deviation of economies of scope with a Taylor expansion but keeping only the first two terms of the Taylor series. However, the Taylor approximation is non-linear in the estimated parameters. Further, it could produce inaccurate estimates if higher-order terms of Taylor expansion are important. Another approximate approach is bootstrap, a data-based simulation method for statistical inference proposed by Efron (1979) and first applied to construct confidence intervals for economies of scope by Eakin, McMillen, \& Buono (1990). Bootstrap uses resampling technique to construct the confidence intervals that are functions of parameter estimates like economies of scope. However, either delta method or bootstrap still neglects the uncertainty of regression coefficients since it still treats estimated coefficients fixed during the process. The misleading results could be made due to the underestimated prediction variance.

The preceding problems could be more appropriately treated with a Bayesian approach that is always able to produce probability distributions for prediction. All desired quantities including the point estimate of economies of scope and its confidence interval are estimated through the use of the posterior distribution that probabilistically integrates the likelihood and the prior information.

In this paper, we develop a Bayesian approach to calculate the point estimate and construct the confidence interval for economies of scope. The remainder of this paper is organized into the following sections. We begin with the short introduction of economies of scope and how they are usually gained. We then propose a Bayesian approach as an alternative method. Section 4 uses a dataset composing of Australian universities to estimate the economies of scope. Finally, we offer our suggestions in the final section.

\section{INFERENCE OF ECONOMIES OF SCOPE}

Let $y=\left(y_{1}, \ldots, y_{n}\right)$ denote $n$ types of product of a firm and $w=\left(w_{1}, \ldots, w_{m}\right)$ denote $m$ types of input prices. Economies of scope are defined over the entire output set $N=(1, \ldots, n)$ whose output quantities are $y=$ $\left(y_{1}, \ldots, y_{n}\right)$. These quantities also denote a nontrivial partition, which indicates $\cup_{i} y_{i}=y, y_{i} \cap y_{j}=$ 
Zhang et al, Using a Bayesian approach to evaluate the accuracy of economies of scope: Examples from Australian public universities

$\emptyset$, for $i \neq j, y_{i} \neq \emptyset$, and $n>1$. Therefore, we can define a multiproduct minimum cost function as $C(y, w)$. Economies of scope exist at outputs $y$ and input prices $w$ with respective to the partition $i$ if

$\sum_{i=1}^{n} C\left(y_{i}, w\right)>C(y, w)$

Therefore, (1) shows the total cost of separate production (i.e., the summation of all cost of separate production for $i=1, \ldots, n)$ are larger than the cost of joint production. On the contrary, if the above equality is reversed, it indicates there are diseconomies of scope.

In practice, to know whether there are scope economies, Willig (Willig, 1979) defines the degree of economies of scope (SE) by modifying (1) as

$S E=\frac{\sum_{i=1}^{n} C\left(y_{i}, w\right)-C(y, w)}{C(y, w)}$

In this way, we can gain the point estimate of economies of scope. This equation is also more meaningful: it represents the proportion of cost savings from joint production.

Unfortunately, either costs of separate production or costs of joint production can be directly observed from the data and they are usually estimated from the cost function $C(y, w)$. Therefore, we need to estimate cost function first to calculate the degree of economies of scope. In order to make our model more explicit for each firm, we further stack our observations (cost, outputs and prices) over time for each individual firm. Considering a panel data set, there are $T$ obervations on each of the $n$ individual firms

$C_{f}=C(y, w)+\varepsilon_{f}=Y_{f} \beta+\varepsilon_{f}$, for $f=1 \ldots n$, with $\varepsilon_{f} \sim d n o r m\left(0, \mathrm{I}_{T} \tau_{C}\right)$

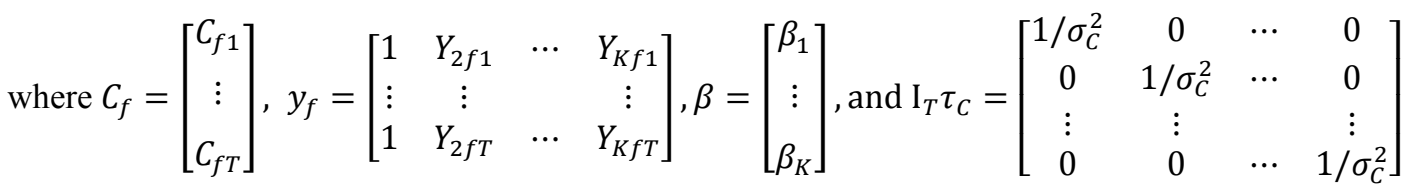

We can see that all coefficients, $\beta$ (including intercepts), are included in a $K$ vector of coefficients, $C_{f}$ to be a $T \times 1$ vector containing $T$ different points in time and $Y_{f}$ which includes output and price vector to be a $T \times K$ matrix containing $T$ different points in time on each of the $K$ explanatory variables. In other words, our observations (cost, outputs and prices) over time have been stacked for each firm $(f)$.

Suppose that the cost function $C(y, w)$ has been estimated with (3) and we can gain $\mathrm{K}$ estimated coefficients $\widehat{\beta_{1}}, \widehat{\beta_{2}}, \ldots, \widehat{\beta_{K}}$. Therefore, SE in (2) is the combinations of mean costs at the point $\mathrm{y}$ and $w$. In practice, the output and price vector will be fixed at their means or the value of $f$-th firm when estimating SE. Since output and price vectors have been fixed at certain level, SE can be equal to a conditional expectation (or mean) function of $K$ estimated parameters, that is,

$\mathrm{SE}=\frac{\sum_{i=1}^{n} E\left(C \mid y_{i}, w, y_{j}=0 \text { for } j \neq i\right)-E(C \mid y, \mathrm{w})}{E(C \mid y, \mathrm{w})}=\operatorname{SE}(\widehat{\beta})$

where the hat symbol ${ }^{-}$indicates quantities estimated from the observed data and $E$ denotes the expected value of cost.

\section{BAYESIAN INFERENCE}

From a frequentist view, SE is assumed fixed but unknown. Inferences about SE are based on the distribution of data statistics under repeated sampling. In contrast, from a Bayesian view, SE is considered to be a random variable and summarized in a probability distribution that will be derived in the following section.

Following the setting of (3), $C_{f}$ has a normal distribution (dnorm), and then the likelihood function for $n$ multiple linear regressions on $K$ variables with balanced panel data could be derived as follows:

$C_{f} \mid y_{f}, \beta, \tau_{C} \sim \operatorname{dnorm}\left(y_{f} \beta, \mathrm{I}_{T} \tau_{C}\right)$, for $f=1 \ldots n$ 
Zhang et al, Using a Bayesian approach to evaluate the accuracy of economies of scope: Examples from Australian public universities

To conduct a Bayesian approach, we need to assign prior information to each estimated parameters $\left(\beta, \tau_{C}\right)$. We start with the variance of $\operatorname{cost} \tau_{C}$. Without prior information on the variance, a gamma distribution with lower value of shape and scale parameters is a popular non-informative prior for $\tau_{C}$.

$\tau_{C} \mid a, b \sim \operatorname{dgamma}(a, b)$

We further assume that all coefficients are drawn from the normal distribution (dnorm):

$\beta \mid \mathrm{c}, \mathrm{d} \sim \operatorname{dnorm}(c, d)$

With the prior and likelihood function, we can use Bayes' theorem to derive the posterior density function that is proportional to $(\propto)$ the likelihood function and prior distribution.

$p\left(\theta \mid C_{f}\right) \propto p\left(C_{f} \mid \theta\right) p(\theta)=\left[\prod_{f=1}^{N} p\left(C_{f} \mid y_{f}, \beta, \tau_{C}\right)\right] p(\beta \mid \mathrm{c}, \mathrm{d}) p\left(\tau_{C} \mid a, b\right)$

where $\theta \equiv\left[\beta, \tau_{C}\right]$ includes all the parameters of our model.

This posterior density function is conditional on the observed costs, $C_{f}$ and is also the joint posterior density for all the parameters of our model. The marginal posterior distribution for each parameter could be derived by integrating other parameters out of (8). Then this distribution would be further used for the inferences of each parameter.

With posterior density function in hand, we can apply our proceeding analysis to (4) and predict the corresponding degree of economies of scope, $S E(\beta)$.

$p\left(S E(\beta) \mid C_{f}\right)=\int p\left(S E(\beta) \mid C_{f}, \theta\right) p\left(\theta \mid C_{f}\right) d \theta$

Here, $p\left(S E(\beta) \mid C_{f}, \theta\right)$ is the sampling distribution of future cost estimates. That is, we consider degree of economies of scope as an observation predicted by the estimated cost function. This predicted value is another dataset generated by the same model and therefore will still have a normal distribution with a mean $E S S_{S E}$ and the same inverse variance $\left(\tau_{C}\right)$. In a Bayesian framework, the prediction is carried out by sampling from the posterior predictive distribution as follows.

$S E(\beta) \mid C_{f} \sim \operatorname{dnorm}\left(E S S_{S E}, \tau_{C}\right)$

where $E S S_{S E}$, is derived from using different output mean values.

Intervals for an interest parameter like the degree of economies of scope could be constructed in a common probability structure (Casella \& Berger, 2002):

$\operatorname{Prob}(L \leq \mathrm{SE} \leq U)=1-\alpha$

where $L$ and $U$ are the lower and upper interval limits, $\alpha$ is significance level, and $1-\alpha$ is confidence level. Its Bayesian confidence interval is therefore determined via

$\int_{L}^{U} p\left(S E(\beta) \mid C_{f}\right) d S E(\beta)=1-\alpha$

where $p\left(S E(\beta) \mid C_{f}\right)$ is the posterior distribution of $S E(\beta)$ conditioned on observed cost $C_{f}$ derived in (9)

One additional advantage of Bayesian approach is that we can further calculate the probability of existence of economies of scope:

$\operatorname{Prob}\left(\operatorname{SE}(\beta)>0 \mid C_{f}\right)$

This is evaluated with respect to the posterior predictive distribution in (10). It is worth noting that for those studies that gave the conclusion based on the only point estimate of scope economies, they implicitly assume equation (13) is equal to $100 \%$.

\section{EMPIRICAL APPLICATION TO AUSTRALIAN PUBLIC UNIVERSITIES}

To make our demonstration easy to understand, we limit the number of outputs and firms in the production process to demonstrate the model clearly in the preceding section. We also assume the price is constant across the firms to avoid the regularity conditions such as linearly homogenous, non-decreasing, and concave 
Zhang et al, Using a Bayesian approach to evaluate the accuracy of economies of scope: Examples from Australian public universities

in input prices. In this way, we can focus on how to calculate the economic estimates through a Bayesian approach and compare it with other two methods. These conditions lead us to use the dataset from higher education institutions whose main outputs could be reduced to two, that is, teaching and research (Cohn \& Cooper, 2004). The assumption of constant price is also reasonable because institutions are usually in the highly regulated contexts (Agasisti \& Johnes, 2010).

The Australian university Table 1. Descriptive Statistics of the data over ten years (2003-2012)

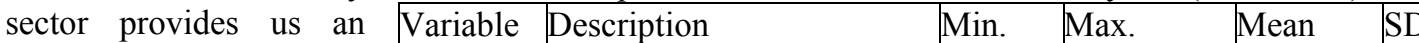
appropriate data set for our model demonstration. This sector has experienced significant

\begin{tabular}{|l|l|l|l|l|l|}
\hline Variable & Description & Min. & Max. & Mean & SD \\
\hline$C$ & Total cost ('000 AUD) & 34,355 & $1,327,778$ & 400,671 & 280,776 \\
\hline$y_{1}$ & No. of student completions & 546 & 17,085 & 6,602 & 3,551 \\
\hline$y_{2}$ & No. of publications & 60.76 & 5,118 & 1,222 & 1,087 \\
\hline
\end{tabular}

changes for the last 30 years. It used to contain many small and specialized higher education institutions but it is no longer the case. Universities are given more responsibilities for enrolling more students and raising their financial revenues (Bradley, 2008). We employ a balanced panel dataset composed of whole 37 Australian public universities over the years 2003-2012 that is the longest periods for public access ${ }^{1}$. These are totally 370 annual observations obtaining from the Australian Government Department of Education. The monetary variable, total cost (total expenses from continuing operations) has been converted to real values (year $2003=100$ ) with a consumer price index of Australian Bureau of Statistics. Two outputs are included in the cost function. Outputs $\left(y_{i}\right)$ consists of total completions $\left(y_{1}\right)$, and number of publications $\left(y_{2}\right)$. To associate the production cost with outputs and other variables, a proper function form should be chose to approximate the true cost function $C(y, w)$. Baumol et al.(1982) have suggested three multi-product cost functional forms, Constant Elasticity of Substitution (CES), Hybrid Translog (TL) function, and Quadratic Cost Function (QCF). Each form makes the measurement of economies of scope possible and allows returns to scale to vary. These functional forms also correspond to the ideal conditions of cost functional form: flexible enough to allow scale and economies of scope to vary with the levels of output, allow data to decide the existence of scale and scope economics, and permit zero outputs of some services (Lloyd, Morgan, \& Williams, 1993). The quadratic cost function is preferred in the estimation of economies of scope since this cost function permits an output to have zero values without further transformation like other forms.

With our panel data set, we can modify (3) into

$$
\begin{aligned}
C_{f t}= & \beta_{0}+\beta_{1} y_{1 f t}+\beta_{2} y_{2 f t}+0.5\left(\beta_{11}\left(y_{1 f t}\right)^{2}+\beta_{22}\left(y_{2 f t}\right)^{2}\right)+\beta_{12}\left(y_{1 f t}\right)\left(y_{2 f t}\right)+\varepsilon_{f t} \\
& \text { for } f=1 \ldots 37 \text { and } t=1 \ldots 10
\end{aligned}
$$

All the notations follow the previous sections. We can notice that the number of variables, $K$, is 6 $\left(\beta_{k, k=\{0,1,2,11,22,12\}}\right)$. Our model is fitted using the following non-informative priors. These choices could not only give posterior estimates similar to maximum likelihood point estimates but also offer additional uncertainty over estimates of economies of scope as well as the uncertainty of regression coefficients.

Notice that we assume prices are constant across institutions and, therefore, there is no need to include the price variables in the estimation process. We could get the corresponding posterior predictive distribution for the estimate of economies of scope as follows.

Table 2. Priors used in BUGS
\begin{tabular}{|l|l|}
\hline Parameters & Priors \\
\hline$\beta_{k}$ & dnorm $(0,0.000001)$ \\
\hline$\tau_{c}$ & dgamma $(0.1,0.01)$ \\
\hline
\end{tabular}

$S E(\beta) \mid C_{f t} \sim \operatorname{dnorm}\left(E S S_{S E}, \tau_{C}\right)$

where

$$
E S S_{S E}=\frac{\beta_{0}-\beta_{12} y_{1} y_{2}}{\beta_{0}+\beta_{1} y_{1}+\beta_{2} y_{2}+0.5\left(\beta_{11}\left(y_{1}\right)^{2}+\beta_{22}\left(y_{2}\right)^{2}\right)+\beta_{12} y_{1} y_{2}}
$$

There is no analytical solution for the posterior density function above but a Gibbs sampler could be used to derive the estimates of the unknown parameters with the prior knowledge (Gelfand, Hills, Racine-Poon, \& Smith, 1990). These estimates will be derived with statistical software, BUGS (Bayesian inference using Gibbs sampling) (Lunn, Spiegelhalter, Thomas, \& Best, 2009) and it will choose a proper Markov Chain Monte Carlo (MCMC) sampling algorithm based on the model structure we specified above. All inferences

\footnotetext{
${ }^{1}$ The data set could be downloaded from http://docs.education.gov.au/ .
} 
Zhang et al, Using a Bayesian approach to evaluate the accuracy of economies of scope: Examples from Australian public universities

are tested by performing two parallel chains running for 50,000 with a burn-in of first 25,000 iterations. The convergence diagnostic indicator, potential scale reduction $(\hat{R})$ (Brooks \& Gelman, 1998), is used to monitor the convergence. This unbiased estimator (close to one as the number of draws approach infinity) also has the advantage of reliability compared with purely using plots. As Table 3 shows, all the estimated parameters are obtained from our Bayesian approach. We obtained $\hat{R}<1.04$ for all estimates, indicating good convergence.

For a significance level of $\alpha$, a frequentist confidence interval with a $100(1-\alpha) \%$ confidence interval is an interval to be expected to cover the true value of SE $100(1-\alpha) \%$ of the time in the repeated sampling of observations. On the other hand, a Bayesian confidence interval does not rely on repeated sampling but the posterior probability that the SE lies in the interval (Box \& Tiao, 1992). Now, we can use the simulation results to demonstrate the degree of economies of scope with its Bayesian confidence intervals. Choosing a significance level of $5 \%$, we can draw the $95 \%$ confidence intervals for the point estimates evaluated at $50 \%$ to $300 \%$ of output mean in Figure 1. Notice that the positive figure of $\beta_{12}$ in Table 3 implies sufficient condition of diseconomies of scope (Hajargasht, Coelli, \& Rao, 2008; Pulley \& Humphrey, 1993). Negative values of degree of economies of scope are therefore expected. As Figure 1 shows, we can find the consistent results with Table 3: all Bayesian point estimates are negative and its probability of existence of economies of scope produced with (13) is very close to zero ${ }^{2}$. Another interesting finding is that the range of confidence interval increases with the

Table 3. Estimates of Bayesian approach

\begin{tabular}{|l|l|l|}
\hline & Mean & Std. Error \\
\hline$\beta_{0}$ & 510.00 & 4.4760 \\
\hline$\beta_{1}$ & 23.42 & 0.0114 \\
\hline$\beta_{2}$ & 214.3 & 0.0549 \\
\hline$\beta_{11}$ & -0.0016 & 0.0000 \\
\hline$\beta_{22}$ & -0.0261 & 0.0000 \\
\hline$\beta_{12}$ & 0.0056 & 0.0000 \\
\hline
\end{tabular}
expansion of percentage of output mean.

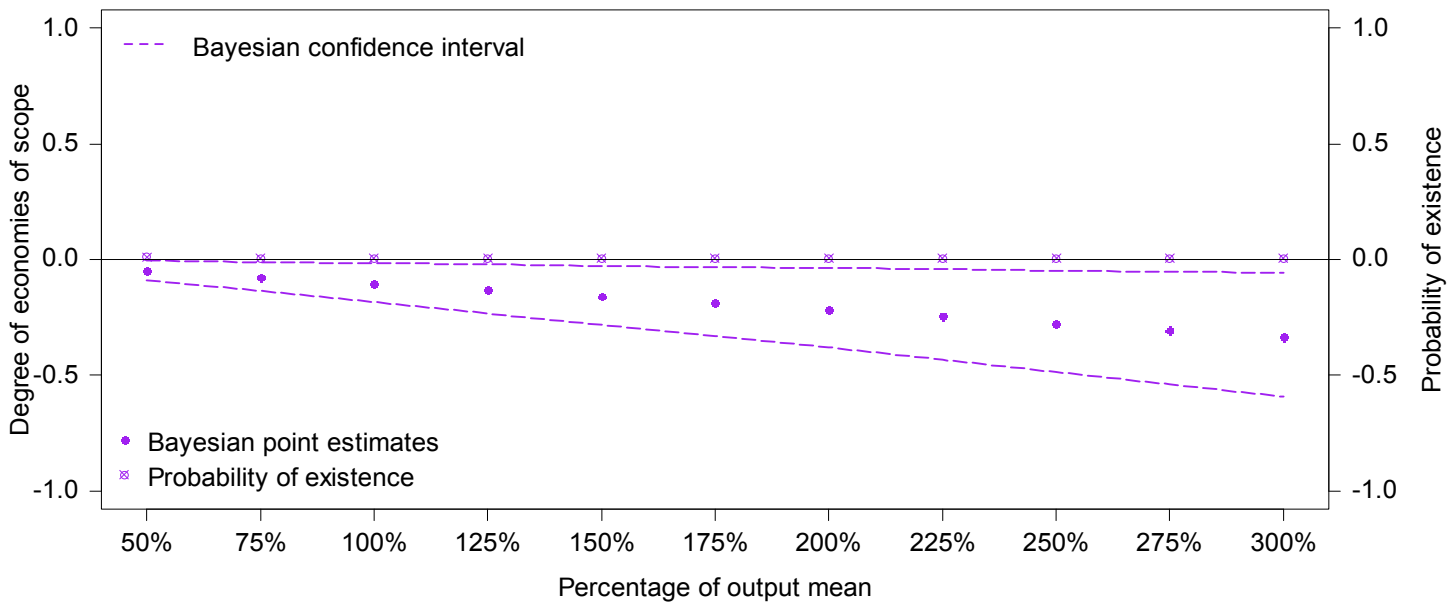

Figure 1. Plot of Bayesian point estimates of economies of scope with Bayesian confidence intervals and its probability of existence of economies of scope.

\section{CONCLUDING REMARKS}

The main purpose of this study is to develop a new approach for estimating inference measures for scope economies. We demonstrate our model without incorporating the prices for the simplicity. Our approach might also be applied to other economic index (such as economies of scale, marginal costs, output-cost elasticities, Allen partial elasticities of input substitution) predicted by a cost function. Further studies especially applications in contexts of the free market should consider the situation without constant prices across firms. In addition, the cost function should obey several rules such as curvature. Fortunately, these restrictions are relatively easy imposed within a Bayesian framework. Another extension comes when researchers have more than one model to predict the economies estimates. Model averaging allows all models to be included in the posterior predictive distribution without deleting any of them.

\section{ACKNOWLEDGMENTS}

The authors thank Dr. Wen-Hsi Yang for providing useful comments to this work.

\footnotetext{
${ }^{2} \mathrm{R}$ and BUGS codes could be downloaded in http://liangchengzhang.weebly.com/publications.html
} 
Zhang et al, Using a Bayesian approach to evaluate the accuracy of economies of scope: Examples from Australian public universities

\section{REFERENCES}

Agasisti, T., \& Johnes, G. (2010). Heterogeneity and the evaluation of efficiency: The case of Italian universities. Applied Economics, 42(11), 1365-1375. doi: 10.1080/00036840701721463

Baumol, W. J., Panzar, J. C., \& Willig, R. D. (1982). Contestable Markets and the Theory of Industry Structure. New York, NY: Harcourt Brace Jovanovich.

Box, G. E. P., \& Tiao, G. C. (1992). Bayesian Inference in Statistical Analysis. New York, NY: John Wiley \& Sons.

Bradley, D. (2008). Review of Australian Higher Education: Final Report. Canberra: Department of Education, Employment and Workplace Relations.

Brooks, S. P., \& Gelman, A. (1998). General methods for monitoring convergence of iterative simulations. Journal of Computational and Graphical Statistics, 7(4), 434-455.

Carey, K., Burgess, J. F., \& Young, G. J. (2015). Economies of scale and scope: the case of specialty hospitals. Contemporary Economic Policy, 33(1), 104-117. doi: 10.1111/coep.12062

Casella, G., \& Berger, R. L. (2002). Statistical Inference (2nd ed.). Pacific Grove, CA: Duxbury

Cohn, E., \& Cooper, S. T. (2004). Multi-product cost functions for universities: economies of scale and scope. In G. Johnes \& J. Johnes (Eds.), International Handbook on the Economics of Education (pp. 579-612). Cheltenham, UK: Edward Elgar Publishing Ltd.

Delgado, M. S., Parmeter, C. F., Hartarska, V., \& Mersland, R. (2015). Should all microfinance institutions mobilize microsavings? Evidence from economies of scope. Empirical Economics, 48(1), 193-225. doi: 10.1007/s00181-014-0861-3

Eakin, B. K., McMillen, D. P., \& Buono, M. J. (1990). Constructing confidence intervals using the bootstrap: An application to a multi-product cost function. The Review of Economics and Statistics, 72(2), 339344. doi: $10.2307 / 2109725$

Efron, B. (1979). Bootstrap methods: another look at the jackknife. The Annals of Statistics, 1-26.

Gelfand, A. E., Hills, S. E., Racine-Poon, A., \& Smith, A. F. M. (1990). Illustration of Bayesian inference in normal data models using Gibbs sampling. Journal of the American Statistical Association, 85(412), 972-985. doi: $10.2307 / 2289594$

Hajargasht, G., Coelli, T., \& Rao, D. S. (2008). A dual measure of economies of scope. Economics Letters, 100(2), 185-188. doi: http://dx.doi.org/10.1016/j.econlet.2008.01.004

Lloyd, P., Morgan, M., \& Williams, R. (1993). Amalgamations of universities: Are there economics of scale or scope? Applied Economics, 25(8), 1081-1092.

Lunn, D., Spiegelhalter, D., Thomas, A., \& Best, N. (2009). The BUGS project: evolution, critique and future directions. Statistics in medicine, 28(25), 3049-3067.

Meyer, R. (2012). Vertical economies and the costs of separating electricity supply: a review of theoretical and empirical literature. Energy Journal, 33(4), 161-185.

Ohe, Y., \& Kurihara, S. (2013). Evaluating the complementary relationship between local brand farm products and rural tourism: evidence from Japan. Tourism Management, 35, 278-283.

Panzar, J. C., \& Willig, R. D. (1981). Economies of scope. The American Economic Review, 71(2), $268-272$.

Pulley, L. B., \& Humphrey, D. B. (1993). The role of fixed costs and cost complementarities in determining scope economies and the cost of narrow banking proposals. The Journal of Business, 66(3), 437462. doi: $10.2307 / 2353208$

Saal, D. S., Arocena, P., Maziotis, A., \& Triebs, T. (2013). Scale and scope economies and the efficient vertical and horizontal configuration of the water industry: a survey of the literature. Review of Network Economics, 12(1), 93-129.

Willig, R. D. (1979). Multiproduct technology and market structure. The American Economic Review, 69(2), 346-351. doi: 10.2307/1801671

Worthington, A. C., \& Higgs, H. (2011). Economies of scale and scope in Australian higher education. Higher Education, 61(4), 387-414. doi: 10.1007/s10734-010-9337-3 